\title{
3.5 Arterial Stiffness Partly Explains Sex Differences in Associations Between Left Ventricular Structure and Mortality: The Southall and Brent Revisited (SABRE) Study
}

\author{
Lamia Al Saikhan ${ }^{1, *}$, Chloe Park ${ }^{1}$, Therese Tillin ${ }^{1}$, Jamil Mayet ${ }^{2}$, Nish Chaturvedi $^{1}$, Alun Hughes ${ }^{1}$ \\ ${ }^{1}$ University College London (UCL), London, UK \\ ${ }^{2}$ Imperial College London and Imperial College Healthcare NHS Trust
}

\section{ABSTRACT}

Background: We have previously shown that 3D echocardiography-derived (3DE) indices of left ventricular (LV) structure predict all-cause mortality differently in men and women [1]. However, the mechanism behind these sex differences remains unknown. We therefore examined the role of macro- and micro-vascular disease in explaining these sex differences.

Methods: 910 individuals (age, $69.7 \pm 6.2$ years; $77.7 \%$ male) from the SABRE study, a UK-largest tri-ethnic longitudinal cohort, underwent a health examination. Associations between 3DE-derived LV measures (end-diastolic volume (EDV), end-systolic volume (ESV) and LV remodeling index (LVRI)) and all-cause mortality were determined using multivariable Cox regression. Total arterial compliance (TAC), augmentation index (AI), eGFR and microalbuminuria were added to these models.

Results: 103 (14.5\%) men and 17 (8.4\%) women died over median follow-up of 8 years. After multivariable adjustment, lower LVRI, and higher volumes were independently associated with increased risk of mortality in men; however, associations were reversed in women (Table $1 ; p$ (interaction: LVRI $=0.034$, EDV $=0.051$, and ESV $=0.009$ )). TAC was lower in women $(0.73 \pm 0.24$ vs $0.83 \pm 0.27, p<0.0001)$ and AI higher $(156.1 \pm 22.3$ vs $145.4 \pm 21, p<0.0001$; adjustment for TAC and AI attenuated the associations in women, but not in men (Table 1), resulting in smaller sex differences in associations. Adjustment for measures of microvascular disease had negligible effect on associations in men or women.

Conclusion: In a large tri-ethnic sample of the UK general population, sex differences in associations between 3DE LV structural measures and mortality seem to be partially explained by arterial stiffness and wave reflection.

Table 1 Associations between 3DE-derived indices of LV structure and all-cause mortality

\begin{tabular}{|c|c|c|}
\hline & Men, HR (95\% CI), $p$ & Women, HR (95\% CI), $p$ \\
\hline 3D LVRI ${ }^{\dagger}$ & $(N=689 ; 99$ deaths, $\mathrm{SD}=0.37 \mathrm{~g} / \mathrm{ml})$ & $(N=190 ; 16$ deaths, $\mathrm{SD}=0.34 \mathrm{~g} / \mathrm{ml})$ \\
\hline Model 1 & $0.77(0.63,0.94), \mathbf{0 . 0 1 2}$ & $1.5(0.95,2.5), 0.082$ \\
\hline + TAC & $0.82(0.66,1.0), 0.071$ & $1.25(0.72,2.1), 0.426$ \\
\hline + Central AI & $0.82(0.67,1.0), 0.072(N=629 ; 89$ deaths $)$ & $1.23(0.67,2.2), 0.504(N=170 ; 13$ deaths $)$ \\
\hline + TAC + Central AI & $0.87(0.70,1.1), 0.260(N=629 ; 89$ deaths $)$ & $1.1(0.58,2.0), 0.801(N=170 ; 13$ deaths $)$ \\
\hline+ eGFR & $0.79(0.64,0.96), \mathbf{0 . 0 2 1}$ & $1.5(0.95,2.4), 0.083$ \\
\hline + Microalbuminuria & $0.78(0.64,0.95), \mathbf{0 . 0 1 5}$ & $1.6(0.99,2.8), 0.053$ \\
\hline$+\mathrm{eGFR}+$ Microalbuminuria & $0.79(0.65,0.97), \mathbf{0 . 0 2 3}$ & $1.6(0.98,2.7), 0.061$ \\
\hline 3D EDV & $\left(N=707 ; 103\right.$ deaths, $\left.S D=9.9 \mathrm{ml} / \mathrm{m}^{2}\right)$ & $\left(N=203 ; 17\right.$ deaths, $\left.\mathrm{SD}=7.3 \mathrm{ml} / \mathrm{m}^{2}\right)$ \\
\hline Model 1 & $1.26(1.1,1.5), 0.007$ & $0.51(0.25,1.1), 0.076$ \\
\hline$+\mathrm{TAC}$ & $1.23(0.99,1.5), 0.060$ & $0.78(0.29,2.0), 0.613$ \\
\hline+ Central AI & $1.25(1.04,1.5), 0.013(N=646 ; 92$ deaths $)$ & $0.75(0.31,1.8), 0.519(N=183 ; 14$ deaths $)$ \\
\hline$+\mathrm{TAC}+$ Central AI & $1.22(0.98,1.5), 0.078(N=646 ; 92$ deaths $)$ & $1.0(0.38,3.1), 0.871(N=183 ; 14$ deaths $)$ \\
\hline + eGFR & $1.23(1.0,1.45), \mathbf{0 . 0 1 4}$ & $0.51(0.25,1.0), 0.071$ \\
\hline + Microalbuminuria & $1.25(1.0,1.5), \mathbf{0 . 0 1 0}$ & $0.60(0.28,1.3), 0.189$ \\
\hline+ eGFR + Microalbuminuria & $1.23(1.0,1.45), 0.016$ & $0.57(0.27,1.2), 0.144$ \\
\hline 3D ESV & $\left(N=707 ; 103\right.$ deaths, $\left.\mathrm{SD}=6.1 \mathrm{ml} / \mathrm{m}^{2}\right)$ & $\left(N=203 ; 17\right.$ deaths, $\left.\mathrm{SD}=3.7 \mathrm{ml} / \mathrm{m}^{2}\right)$ \\
\hline Model 1 & $1.37(1.14,1.66), \mathbf{0 . 0 0 1}$ & $0.56(0.31,1.0), \mathbf{0 . 0 4 8}$ \\
\hline$+\mathrm{TAC}$ & $1.34(1.1,1.6), 0.004$ & $0.68(0.36,1.3), 0.221$ \\
\hline+ Central AI & $1.38(1.13,1.7), \mathbf{0 . 0 0 1}(N=646 ; 92$ deaths $)$ & $0.72(0.36,1.4), 0.347(N=183 ; 14$ deaths $)$ \\
\hline$+\mathrm{TAC}+$ Central AI & $1.35(1.1,1.7), 0.004(N=646 ; 92$ deaths $)$ & $0.82(0.40,1.7), 0.598(N=183 ; 14$ deaths $)$ \\
\hline
\end{tabular}




\begin{tabular}{|c|c|c|}
\hline 3D ESV & $\left(N=707 ; 103\right.$ deaths, $\left.\mathrm{SD}=6.1 \mathrm{ml} / \mathrm{m}^{2}\right)$ & $\left(N=203 ; 17\right.$ deaths, $\left.\mathrm{SD}=3.7 \mathrm{ml} / \mathrm{m}^{2}\right)$ \\
\hline + eGFR & $1.34(1.1,1.6), 0.002$ & $0.58(0.32,1.0), 0.059$ \\
\hline + Microalbuminuria & $1.37(1.1,1.6), 0.001$ & $0.58(0.31,1.1), 0.079$ \\
\hline + eGFR + Microalbuminuria & $1.33(1.1,1.6), \mathbf{0 . 0 0 2}$ & $0.61(0.33,1.1), 0.104$ \\
\hline
\end{tabular}

Model 1: adjusted for age, ethnicity, SBP, antihypertensive medication, cholesterol: HDL ratio, BMI, DM, smoking. ${ }^{\dagger} \mathrm{LV}$ remodelling index (LVRI) defined as LV mass to end-diastolic volumes (EDV) ratio (the lower numbers for LVRI due to lower available numbers of LV mass $(N=879)) .3 \mathrm{D} \mathrm{ESV}$ is $\log$ transformed. AI, augmentation index (\%); EDV, end-diastolic volumes $\left(\mathrm{ml} / \mathrm{m}^{2}\right)$; eGFR, estimated glomerular filtration rate $\left(\mathrm{ml} / \mathrm{min} / 1.73 \mathrm{~m}^{2}\right)$; ESV, end-systolic volumes $\left(\mathrm{ml} / \mathrm{m}^{2}\right)$; HR, hazard ratio; LVRI, left ventricular remodelling index $(\mathrm{g} / \mathrm{ml})$; TAC, total arterial compliance ( $\mathrm{ml} / \mathrm{mmHg})$; SD, standard deviation.

\section{REFERENCE}

[1] Al Saikhan L, Park C, Tillin T, Mayet J, Chaturvedi N, Hughes A. 3 3D echocardiography-derived indices of left ventricular function and structure predict long-term mortality differently in men and women: the Southall And Brent Revisited (SABRE) study. Heart 2019;105:A3-A5.

(c) 2019 Association for Research into Arterial Structure and Physiology. Publishing services by Atlantis Press International B.V. This is an open access article distributed under the CC BY-NC 4.0 license (http://creativecommons.org/licenses/by-nc/4.0/). 